\title{
An updated scoring system for prediction of survival after resection of colorectal liver metastases: addition of KRAS status as an important risk modifier
}

\author{
Evan Jost, Don Major, Elijah Dixon \\ Department of Hepatobiliary Surgery, University of Calgary, Calgary, Canada \\ Correspondence to: Elijah Dixon. Department of Hepatobiliary Surgery, University of Calgary, Calgary, Canada. Email: elijah.dixon@albertahealthservices.ca. \\ Comment on: Brudvik KW, Jones RP, Giuliante F, et al. RAS mutation clinical risk score to predict survival after resection of colorectal liver \\ metastases. Ann Surg 2019;269:120-6.
}

Submitted Jun 04, 2019. Accepted for publication Jun 11, 2019.

doi: $10.21037 /$ hbsn.2019.06.06

View this article at: http://dx.doi.org/10.21037/hbsn.2019.06.06

Colorectal liver metastases (CRLM) were traditionally associated with a very poor prognosis after resection, with some historical series reporting 5 -year survival rates as low as $14 \%$ for completely resected multiple metastases (1). However, as modern chemotherapy and surgical techniques have evolved, there has been much progress made in improving survival for this unique group of patients, with 5 -year survival approaching 55\% with R0 resection (2). However, not all CRLM are the same, with very different biological behaviour and ultimately oncologic outcomes in different patients. There have been many attempts to create a scoring system defining factors which will predict this behaviour, with the system created by Fong et al. in 1999 being the most commonly employed (3). These scoring systems are important because they help the selection of patients who are likely to benefit from surgical treatment of their metastases. Patients who are unlikely to benefit from surgical resection may be better treated by alternative and potentially less morbid therapies, such as the various forms of ablation, or with systemic treatments. It is apparent that the accuracy of this prediction is essential to allow patients to benefit either by having appropriate surgical therapy or by avoiding potentially unnecessary surgical morbidity.

RAS is a downstream signalling molecule in the EGFR pathway. RAS mutations are present in $15-36 \%$ of patients with CRLM. They have been shown to be prognostic of both recurrence risk and overall survival (OS), and RAS mutational status is the only molecular marker currently approved for this purpose (2). KRAS mutations have been found to confer a HR for death of 2.0 compare to KRAS wildtype tumours, with a 5 -year OS after resection of $23 \%$ compared to $61 \%$ for patients with wildtype tumours (2). RAS mutations have also been associated with decreased radiologic and pathologic response to neoadjuvant chemotherapy regimens such as FOLFOX and Bevacizumab combination therapy. A study performed at the University of Texas showed a pathologic response rate of $58.9 \%$ in RAS-wildtype patients versus $36.8 \%$ in patients with RASmutant tumors (4). This also translated into worse overall and recurrence-free survival (RFS) in the same study.

These observations prompted the authors of the study published by Brudvik and colleagues in Annals of Surgery in 2017 to question the current applicability of the MSKCC clinical risk score to modern patients with resectable CRLM (5), given that the MSKCC score was derived from patients treated from 1985 to 1998 . This scoring system incorporates the size of the largest metastasis, the number of metastases, CEA level, nodal status of the primary, and disease-free interval less than 12 months (3). They further hypothesized that the addition of RAS mutational status would further add to the accuracy of this score given its status as an independent strong predictor of survival, response to therapy, and recurrence.

Brudvik et al. then performed a multivariate analysis using the original scoring system on a modern cohort of patients whose RAS mutation status was known. They found that only primary nodal status, diameter of the largest metastasis, and RAS mutation status were associated with OS. They therefore combined these factors into a modified scoring system which they termed the m-CS, which 
predicted overall and RFS significantly more accurately than the original MSKCC score from which it was derived. The m-CS and t-CS (MSKCC score) showed c-statistics of 0.69 (95\% CI, 0.62-0.76) and 0.57 (95\% CI, 0.48-0.65) respectively. They further showed that the addition of each point on the m-CS was associated with a corresponding decrease in OS and RFS, a finding which did not hold true for the original score. These findings were then validated using an international multicentre validation cohort with similar results, with the caveat that there were differences in survival and recurrence between centres in the cohort. Additionally, the authors provided evidence that the difference was due to the addition of RAS status, rather than simply the reduction in numbers of variables included in the score.

This is a timely and well-conducted study which has several strengths. The first and potentially most important of these is that the authors provide an assessment of the currently most widely used clinical scoring system in a group of modern patients. The second is that they have provided a clinically useful way to add RAS status to our overall risk assessment for recurrence and survival after CRLM resection. Given that RAS status is an independent predictor of OS and RFS, it is likely important to include in our preoperative assessment of risk and clinical utility. The third strength of this new scoring system is that it is even simpler than the previous t-CS. Simplification and greater ability to stratify risk may make this scoring system more pragmatically useful in everyday clinical settings.

There were several questions raised by the study, particularly with respect to patient selection. The current study was understandably limited to patients with known RAS mutational status. The study included patients from 2005 to 2013. The use of RAS mutational status to select patients for cetuximab therapy was approved by the FDA in the US in 2012 (6). The indications used by the centre to select patients for RAS testing prior to this period were therefore unclear, and not stated in the article. This may introduce some bias in the results of the study, given that these patients may have had a different prognosis than other patients with CRLM during the study period. This may limit the generalizability of the results.

There was also some variability in the use of preoperative systemic therapies by the centre during the study. Some of this may have been due to evolution of therapies during the study, but there were also variations in the length of treatment preoperatively. Similarly, the use of postoperative systemic therapies was not included in the analysis. This variation in systemic therapy could also introduce bias to the study, given the effect that these agents have on survival and recurrence in other patient cohorts (7).

Taken together, these results have provided strong evidence that RAS status can be used in combination with other factors as a strong predictor of both overall and RFS after the resection of CRLM. Brudvik and colleagues have provided a simple and clinically-relevant means of assessing risk and, in part, suitability for surgical treatment for patients with CRLM. This is particularly important given its evaluation in a modern and international cohort of patients which speaks to its external validity. The authors have therefore made a number of important contributions to the preoperative evaluation of patients with CRLM in the context of modern multidisciplinary care. This is just the start of highly individualized cancer care-the future promises further incremental improvements in patient care.

\section{Acknowledgments}

None.

\section{Footnote}

Conflicts of Interest: The authors have no conflicts of interest to declare.

Ethical Statement: The authors are accountable for all aspects of the work in ensuring that questions related to the accuracy or integrity of any part of the work are appropriately investigated and resolved.

\section{References}

1. Cady B, McDermott WV. Major hepatic resection for metachronous metastases from colon cancer. Ann Surg 1985;201:204-9.

2. Tsilimigras DI, Ntanasis-Stathopoulos I, Bagante F, et al. Clinical significance and prognostic relevance of KRAS, BRAF, PI3K and TP53 genetic mutation analysis for resectable and unresectable colorectal liver metastases: A systematic review of the current evidence. Surg Oncol 2018;27:280-8.

3. Fong Y, Fortner J, Sun RL, et al. Clinical score for predicting recurrence after hepatic resection for metastatic colorectal cancer: analysis of 1001 consecutive cases. Ann Surg 1999;230:309-18; discussion 318-21.

4. Zimmitti G, Shindoh J, Mise Y, et al. RAS mutations 
predict radiologic and pathologic response in patients treated with chemotherapy before resection of colorectal liver metastases. Ann Surg Oncol 2015;22:834-42.

5. Brudvik KW, Jones RP, Giuliante F, et al. RAS Mutation Clinical Risk Score to Predict Survival After Resection of Colorectal Liver Metastases. Ann Surg 2019;269:120-6.

6. Lieberman R. Food and Drug Administration approval

Cite this article as: Jost E, Major D, Dixon E. An updated scoring system for prediction of survival after resection of colorectal liver metastases: addition of KRAS status as an important risk modifier. Hepatobiliary Surg Nutr 2020;9(1):7072. doi: $10.21037 / \mathrm{hbsn} .2019 .06 .06$ of cetuximab and a new KRAS genetic test for metastatic colorectal cancer: major advance but just the tip of the biomarker iceberg. Am J Ther 2012;19:395-6.

7. Jones RP, Poston GJ. Resection of Liver Metastases in Colorectal Cancer in the Era of Expanding Systemic Therapy. Annu Rev Med 2017;68:183-96. 